\title{
Beta-3-adrenergic receptor Trp64Arg polymorphism: does it modulate the relationship between exercise and percentage of body fat in young adult Japanese males?
}

\author{
Hiroshi Nakashima $\cdot$ Kazuyuki Omae • \\ Tetsuo Nomiyama $\cdot$ Yuko Yamano • \\ Toru Takebayashi $\cdot$ Yutaka Sakurai
}

Received: 21 November 2011 / Accepted: 9 December 2012/Published online: 6 January 2013

(C) The Japanese Society for Hygiene 2013

\begin{abstract}
Objectives The Trp64Arg polymorphism of the beta-3adrenergic receptor gene has been studied as a potential genetic factor contributing to the development of obesity. Several studies have investigated whether or not this polymorphism affects weight reduction due to exercise, but the results of these studies have not been consistent. Moreover, information on a population characterized by a wide ranges of physical activities is scarce. Thus, to further understand the impact of this polymorphism, we examined whether the polymorphism modulates the relationship between physical activity due to exercise (PAE) and percentage of body fat $(\% \mathrm{BF})$.

Methods The study population was 70 Japanese male young adults with a high prevalence of habitual exercise. PAE was estimated by a questionnaire, and \%BF was measured by bioelectrical impedance analysis. Genotyping was done by PCR-restriction fragment length polymorphism analysis.
\end{abstract}

H. Nakashima $(\varangle)$. Y. Sakurai

Department of Preventive Medicine and Public Health,

National Defense Medical College, 3-2 Namiki, Tokorozawa,

Saitama 359-8513, Japan

e-mail: hnakashi@ndmc.ac.jp

K. Omae $\cdot$ T. Takebayashi

Department of Preventive Medicine and Public Health,

School of Medicine, Keio University, Tokyo, Japan

T. Nomiyama

Department of Preventive Medicine and Public Health,

Shinshu University School of Medicine, Matsumoto, Japan

Y. Yamano

Department of Hygiene and Preventive Medicine,

Showa University School of Medicine, Tokyo, Japan
Results The median PAE value of the subjects was 6.9 MET-h/day with an interquartile range of 1.5-10.3 MET-h/ day. PAE correlated significantly with $\% \mathrm{BF}$ in the entire population and within the two subpopulations, namely, carriers or non-carriers of the Arg allele. Multiple regression analysis of PAE, Trp64Arg polymorphism, and the interaction term revealed that while the PAE-derived independent variable was statistically significant, the interaction term was insignificant. When the two regression lines of subjects with and without the Arg allele were considered, the difference between the two slopes did not deviate from zero, nor did the vertical distance of the two regression lines. These findings suggest that the impact of this polymorphism is limited.

Conclusions In our study population of young adult Japanese males, the impact of the Trp64Arg polymorphism on the association between exercise and body composition was weak, if it existed at all.

Keywords Percentage of body fat - Exercise . Beta-3-adrenergic receptor - Polymorphism .

Gene-environment interaction

\section{Introduction}

In 1995 Walston et al. reported that a missense mutation of tryptophan to arginine at codon 64 (Trp64Arg) of the beta3 -adrenergic receptor (ADRB3) gene resulted in subjects with the variant Arg allele having a tendency toward a lower resting metabolic rate [1]. In a subsequent study, carriers of the Arg allele were shown to have a significantly lower basal metabolic rate [2]. As a result of these findings, this polymorphism has been extensively studied as a potential genetic factor contributing to the development of 
obesity, but with inconsistent results [3, 4]. Meta-analyses have therefore been carried out to unify the inconsistent results. A study confined to Japanese people, which is a population with a high Arg allele frequency, revealed that carriers of the Arg allele had a significantly higher body mass index (BMI) than non-carriers, with the difference in BMI being $0.26 \mathrm{~kg} / \mathrm{m}^{2}$ [5]. Thus, the research area is expanding to include the relationship of this polymorphism to hypertension [6].

Reducing excess fat is the target goal for most weightloss intervention programs. Exercise is often recommended or prescribed solely or in combination with diet in these interventions. Because the ADRB3 gene is regarded as one of the genes related to obesity, it is possible that the Trp64Arg polymorphism has an impact on weight reduction due to exercise.

To date, a number of exercise-based intervention studies have addressed this issue [7-9], and several studies featuring both exercise and diet have also been conducted [10-14]. In intervention studies, the amount of exercise is designated in the study design [7-13], and differential weight reduction is studied under the prescribed circumstances. However, the amount of physical activity is usually not great and in some cases is even not clearly stated [14]. These limitations make it difficult to evaluate the effect of the polymorphism on weight reduction induced by a higher level of physical activity. Discrepancies can also be found among the results of these studies. Moreover, continuity after the study period is one of study themes for the intervention study [15].

Because obesity can be defined as the presence of excess body fat, some studies have used the percentage of body fat (\%BF) as an endpoint $[7,12]$. Especially in men, \%BF has been shown to correlate with physical activity-related energy expenditure $[16,17]$ and physical activity measured by a triaxial accelerometer [18]. Therefore, an alternative method to measure the impact of the Arg allele could be an assessment in the presence or absence of the interaction. For example, physical activity, polymorphism and an interaction term may be introduced into the model as a covariate in a multiple regression analysis of \%BF. If a population characterized by a wide range of physical activities is used, it should be feasible to estimate the impact of the Arg allele on individuals with both lower and higher levels of physical activity.

In the study reported here, we examined young adult Japanese males who went to the same university. They were assessed to have negligible levels of energy expenditure due to physical labor and their prevalence of habitual exercise was regarded to be high. Thus, we adapted physical activity due to exercise (PAE) as the surrogate for total activity. We observed the relationship between daily $\mathrm{PAE}$ and \%BF. Interaction of the polymorphism and PAE was tested in three ways: (1) a differential comparison of $\% \mathrm{BF}$ between high and low PAE subjects; (2) multiple linear regression analysis; (3) assessment of the difference between two regression line slopes that were separately generated according to the presence/absence of the Arg allele.

The aims of individuals who are active in sports and exercise are twofold: competition and the maintenance of health. The majority of the population, however, engage in sports or exercise to maintain their health. Thus, the purpose of the study was to determine if the Trp64Arg polymorphism has any effect on the association between body fat and PAE for maintaining health.

\section{Materials and methods}

\section{Study population}

Male students who attended a medical school in Japan were invited to participate in the study. The participants were informed about the study, which included genetic testing, and written informed consent was obtained from each subject. The recruitment of the subjects was conducted in the fall of 1998, and genotyping was completed by June of 1999. Subjects with PAE at the level of an elite athlete were excluded from the analysis. Approval for the study was granted by the Ethical Committee of the National Defense Medical College.

\section{Anthropometric factors}

Information on height was obtained from each participant. Body weight and \%BF were measured by a body fat analyzer based on bioelectrical impedance (TBF-541; Tanita, Tokyo, Japan). We measured the subjects' \%BF at approximately 3 p.m. so that it would not to be affected by diurnal variation. A single examiner measured the waist circumference at the umbilical level and the maximum hip circumference of all participants; this information was used to calculate the waist-to-hip ratio.

Energy intake, alcohol consumption, and smoking status

The subjects were asked to record all the food and beverages they consumed for 7 consecutive days, and each subject's total daily energy intake was estimated based on his self-reported record. Eating patterns that are known risk factors for obesity, such as eating fewer than three meals a day or having a meal prior to going to bed, were also assessed as part of the study. The subjects were asked to list the types of alcoholic beverages they usually consumed in 
order to determine their daily ethanol consumption in grams. Based on their daily ethanol consumption, the subjects were classified into three groups: non-drinker (did not drink or drank only occasionally), light drinker $(<20 \mathrm{~g} /$ day), and moderate to heavy drinker ( $\geq 20 \mathrm{~g} /$ day). A questionnaire on smoking status was also administered, and the subjects were classified into either a non-smoking or a current smoker group.

\section{Estimation of PAE}

The questionnaire asked participants about their habitual exercise patterns. Specifically, subjects were asked to list the types of sports and exercise in which they engaged and the frequency and duration of exercise per week. Based on the information provided in the completed questionnaires, the daily average PAE in MET-h/day was calculated using a textbook on exercise prescription as a reference guide [19].

\section{Genotyping}

Blood was collected for genotyping the Trp64Arg polymorphism in the ADRB3 gene using a PCR-restriction fragment length polymorphism assay. Briefly, genomic DNA was amplified with the primer set 5'-CGC CCA ATA CCG CCA ACA C-3' and 5'-CCA CCA GGA GTC CCA TCA CC-3' [3]. The PCR products were digested with $\mathrm{MvaI}$ at $37{ }^{\circ} \mathrm{C}$ for $3 \mathrm{~h}$, followed by inactivation at $65^{\circ} \mathrm{C}$ for $10 \mathrm{~min}$. The fragments were then separated by agarose gel electrophoresis.

\section{Statistical analysis}

The first step was to examine the characteristics of the study population with respect to the presence/absence of the Arg allele. The normality of variables, including life style and obesity-related indices, was tested, followed by the application of a parametric or non-parametric method, according to the nature of the distribution.

We then verified the correlation between PAE and \%BF in the entire population. The correlation was tested using simple linear regression analysis followed by multivariate analysis with height and weight as covariates.

Interaction of the polymorphism and PAE was tested in three ways. First, we compared the \%BF of the high-PAE subjects to that of the low-PAE subjects. Subjects were classified into two groups according to the median PAE. The \%BFs of the two groups were compared after being stratified by the Trp64Arg polymorphism.

Next, we carried out a multiple regression analysis with an interaction term: $y=b_{0}+b_{1} x_{1}+b_{2} x_{2}+b_{3} x_{1} x_{2}$, where $y$ is $\% \mathrm{BF}$, and $x_{1}$ is a newly designated independent variable calculated by subtracting the median PAE value from that of each subject. $x_{2}$ is a dummy variable representing the Trp64Arg polymorphism. Subjects with the Arg allele were given a 0.5 , and those without the Arg allele were given a -0.5 . These procedures are known as centering. Unless data are centered, the regression coefficient may be irrelevant and misleading [20]. In contrast, when done carefully, centering can diminish almost inevitable multicollinearity. The use of -0.5 and 0.5 is the method for binary data [20]. Subtracting the median value is also recommended for ordinary variables. Particular attention was paid to the statistical significance of $b_{3}$, the partial regression coefficient for the interaction term.

Given two simple linear regression lines $\left(y_{1}=a_{1}+\right.$ $\left.b_{1} x_{1}, y_{2}=a_{2}+b_{2} x_{2}\right)$, one of which is for subjects without the Arg allele and the other for subjects with the Arg allele, the $b_{1}-b_{2} / \mathrm{SE}\left(b_{1}-b_{2}\right)$ statistic follows a $t$ distribution [21]. The difference of two slopes, $b_{1}-b_{2}$ was tested with this statistic. A constant difference might be seen irrespective of the amount of physical activity, when one considers how the Trp64Arg polymorphism affects the relationship between $\% \mathrm{BF}$ and physical activity. When two regression lines are parallel, $b$, the common slope, and $d$, the vertical difference (i.e., the difference in $\% \mathrm{BF}$ ) of the two lines are given as follows: $b=\left(S_{x y 1}+S_{x y 2}\right) /\left(S_{x x 1}+\right.$ $\left.S_{x x 2}\right)$ and $d=\bar{y}_{1}-\bar{y}_{2}-b\left(\bar{x}_{1}-\bar{x}_{2}\right)$ [22]. The $d / \operatorname{SE}(d)$ statistic is known to follow a $t$ distribution. Thus, we additionally calculated $d$, and its statistical significance was tested.

The software package SPSS ver. 16.0J (SPSS Japan, Tokyo, Japan) was partially used for the statistical analyses. A $p$ value of $<0.05$ was considered to be statistically significant.

\section{Results}

The study population consisted of 70 male students ranging in age from 20 to 29 years. Three subjects who were receiving treatment for asthma were excluded. Among the remaining 67 subjects, the software diagnosed two subjects as outliers. One person was playing tennis for $6 \mathrm{~h}$ per day, 7 days per week; the other was playing tennis for $5 \mathrm{~h}$ per day, 7 days per week and jogging for $1 \mathrm{~h}$ per day, 2 days per week. Their PAE levels were apparently comparable to those of elite athletes, and they were excluded, leaving 65 participants as subjects for analysis.

The study population had a high prevalence of habitual exercise. The percentage of subjects who were habitually doing exercise was $84.6 \%$ (55/65 subjects). The median of the daily PAE was $6.9 \mathrm{MET}-\mathrm{h} /$ day [interquartile range (IQR) 1.5-10.3 MET-h/day].

With respect to the ADRB3 genotype, 39 of the subjects were Trp/Trp homozygous, 25 were heterozygous, and one 
Table 1 Characteristics of the study population

\begin{tabular}{|c|c|c|c|}
\hline \multirow[t]{2}{*}{ Study population characteristics } & \multicolumn{2}{|c|}{ Trp64Arg polymorphism } & \multirow[t]{2}{*}{$p$ value } \\
\hline & $\mathrm{T} / \mathrm{T}$ & T/A or $\mathrm{A} / \mathrm{A}$ & \\
\hline$n$ & 39 & 26 & \\
\hline Age (years) & $22(21-22)$ & $22(21-23)$ & $0.744^{\mathrm{a}}$ \\
\hline Height $(\mathrm{cm})$ & $170(166-178)$ & $175(169-177)$ & $0.181^{\mathrm{a}}$ \\
\hline Weight $(\mathrm{kg})$ & $65.1 \pm 1.2$ & $65.0 \pm 1.6$ & $0.948^{\mathrm{b}}$ \\
\hline Daily total energy intake (kcal/day) & $2306 \pm 383$ & $2239 \pm 317$ & $0.466^{\mathrm{b}}$ \\
\hline \multicolumn{4}{|l|}{ Food intake pattern } \\
\hline Eating fewer than 3 times a day (yes/no), $n$ & $8 / 31$ & $6 / 20$ & $1.000^{\mathrm{c}}$ \\
\hline Meal prior to sleep (yes/no), $n$ & $8 / 31$ & $3 / 23$ & $0.503^{\mathrm{d}}$ \\
\hline Alcohol consumption (non-drinker/ $<20 \mathrm{~g} / \geq 20 \mathrm{~g}$ ), $n$ & $17 / 18 / 4$ & $8 / 15 / 3$ & $0.435^{\mathrm{c}, \mathrm{e}}$ \\
\hline Smoking (non-smoker/smoker), $n$ & $33 / 6$ & $25 / 1$ & $0.228^{\mathrm{d}}$ \\
\hline PAE (MET-h/day, mean \pm SD) & $6.9 \pm 0.8$ & $6.2 \pm 1.1$ & $0.615^{\mathrm{b}}$ \\
\hline BMI $\left(\mathrm{kg} / \mathrm{m}^{2}\right)$ & $21.7(20.5-23.4)$ & $21.1(20.1-22.7)$ & $0.312^{\mathrm{a}}$ \\
\hline$\% \mathrm{BF}$ & $16.5(14.5-18.0)$ & $16.8(13.9-20.0)$ & $0.778^{\mathrm{a}}$ \\
\hline Waist-to-hip ratio (\%) & $81.3 \pm 2.9$ & $80.7 \pm 3.8$ & $0.466^{\mathrm{b}}$ \\
\hline
\end{tabular}

$P A E$ Physical activity due to exercise, $B M I$ body mass index, $\% B F$ percentage of body fat

Unless indicated otherwise, data are presented as the median with the interquartile range in parenthesis, or as the mean \pm standard deviation (SD), where appropriate

${ }^{\text {a }}$ Mann-Whitney $U$ test

b Student's $t$ test

${ }^{c}$ Chi-square test

d Fisher's exact test

e Test was performed between non-drinkers and drinkers

was Arg/Arg homozygous. The allele frequency of the Arg allele was 0.208. Because there was only one subject who was homozygous for the Arg allele, a chisquare test for goodness of fit was impossible to conduct. The allele frequency of the study population, however, was comparable to that previously reported in another Japanese population [23]. The one subject who was homozygous for the Arg allele was included in the group of heterozygous subjects for subsequent analyses.

The characteristics of the study population are shown in Table 1. Total daily energy intake, food intake patterns, alcohol consumption, smoking status, and the PAE were comparable between subjects with and without the Arg allele. Additionally, no distinctive differences were observed with regard to BMI, \%BF, or waist-to-hip ratio.

When a simple linear regression analysis was applied to the entire population, PAE was significantly correlated with \%BF (Fig. 1; standardized regression coefficient $-0.361 ; p=0.003)$. Height and weight were found to be good predictors of \%BF in a previous study whose population had a narrow age range similar to ours [18]. In the present study, these variables explained a considerable part of the variation in $\% \mathrm{BF}(F=37.763 ; p<0.001$; adjusted

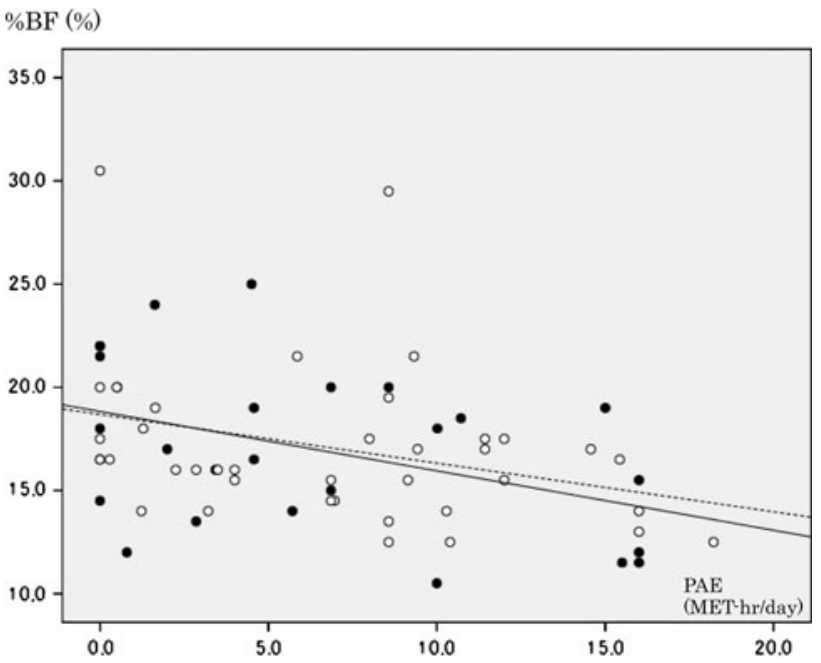

Fig. 1 Scatter plot and regression line of physical activity due to exercise $(P A E)$ and percentage of body fat $(\% B F)$. Open circles Subjects without the Arg allele, filled circles subjects with the Arg allele. Solid line Regression line for subjects with the Arg allele $[y=18.829-0.288 x(r=-0.415, p=0.035)]$, dashed line regression line for subjects without Arg allele $[y=18.684-0.236 x$ $(r=-0.322, p=0.046)]$

$\left.R^{2}=0.535\right)$. Introducing the PAE into the model improved fitness $\left(F=35.650 ; p<0.001\right.$; adjusted $\left.R^{2}=0.619\right)$, as previously demonstrated [18]. 
Table 2 Association between physical activity and the percentage of body fat stratified by the ADRB3 polymorphism

\begin{tabular}{|c|c|c|c|}
\hline \multirow{2}{*}{$\begin{array}{l}\text { Presence/absence } \\
\text { of Arg allele }\end{array}$} & \multicolumn{2}{|c|}{ Percentage of body fat $(\%)$} & \multirow[t]{2}{*}{$p$ value } \\
\hline & Low-PAE group & High-PAE group & \\
\hline Arg allele $(-)$ & $\begin{array}{l}18.1 \pm 3.9 \\
n=17\end{array}$ & $\begin{array}{l}16.3 \pm 3.8 \\
n=22\end{array}$ & $0.059^{\mathrm{a}}$ \\
\hline Arg allele $(+)$ & $\begin{array}{l}18.1 \pm 4.0 \\
n=15\end{array}$ & $\begin{array}{l}15.6 \pm 3.7 \\
n=11\end{array}$ & $0.116^{\mathrm{b}}$ \\
\hline
\end{tabular}

Data are presented as the mean $\pm \mathrm{SD}$

${ }^{\text {a }}$ Tested by the Mann-Whitney $U$ test

b Tested by Student's $t$ test

Table 3 Partial regression coefficients in the multiple regression analysis

\begin{tabular}{lll}
\hline & Standardized partial regression coefficient & $p$ value \\
\hline$b_{1}$ & -0.367 & 0.003 \\
$b_{2}$ & -0.027 & 0.823 \\
$b_{3}$ & -0.036 & 0.763 \\
\hline
\end{tabular}

$y=b_{0}+b_{1} x_{1}+b_{2} x_{2}+b_{3} x_{1} x_{2}$, where $y$ is $\% \mathrm{BF}$, and $x_{1}$ is calculated by subtracting the median PAE value from that of each subject. $x_{2}$ is a dummy variable for the Trp64Arg polymorphism. $x_{1} x_{2}$ is an interaction term

Table 4 Difference in slope and distance between differential regression lines due to polymorphism

\begin{tabular}{llll}
\hline & Difference & $t$ & $p$ value \\
\hline$b_{1}-b_{2}$ & 0.052 & 0.302 & 0.763 \\
$d$ & 0.195 & 0.208 & 0.836 \\
\hline
\end{tabular}

Given two simple linear regression lines $\left(y_{1}=a_{1}+b_{1} x_{1}\right.$, $y_{2}=a_{2}+b_{2} x_{2}$ ), one of which is for subjects without the Arg allele and the other is for subjects with the Arg allele

$b_{1}-b_{2}$, the difference between the two slopes, $d$, the distance between two regression lines in the case of the two regression lines being parallel

Table 2 shows the results of our comparison of the \%BF of the high-PAE subjects and low-PAE subjects. If the polymorphism modulates the relationship, the presence of the Arg allele should attenuate the difference between the low and high groups. However, we observed similar differences between the two groups.

A multiple linear regression analysis was then performed that focused on the statistical significance of the interaction term. While the partial regression coefficient of $x_{1}$, a PAE-derived independent variable, was found to be statistically significant (standardized partial regression coefficient $-0.367, p=0.003$ ), that of $x_{1} x_{2}$, the interaction term, was insignificant (standardized partial regression coefficient $-0.036, p=0.763$ ) (Table 3). The multiple regression analysis revealed a significant main effect of PAE, and a null result was obtained for interaction.

We obtained two simple linear regression lines for subjects with and without the Arg allele, respectively (Fig. 1). The slope of subjects with the variant allele was slightly steeper than that of subjects without the variant allele. The difference between the two slopes was tested to find the null result (Table 4). Then $d$, the vertical difference, and its statistical significance were calculated under the condition of a common slope of -0.258 . The vertical difference (i.e., difference in \%BF) was found not to deviate from zero (Table 4). A null result was observed for vertical difference as well as for the difference in slopes.

These results indicate that the Arg allele had no significant impact on the relationship between PAE and \%BF in our study subjects.

\section{Discussion}

Intervention studies have been conducted to examine the impact of the Trp64Arg polymorphism on weight reduction by exercise. In our study, we used the relationship between physical activity and \%BF and tested whether an interaction exists between physical activity and the ADRB3 gene polymorphism. The characteristic feature of this study is that the relationship between physical activity and body composition was applied to the study of the impact of Trp64Arg on a population characterized by their wide range of PAE.

We found a correlation between PAE and \%BF, which is in line with the results of a previous study [18]. We then examined the impact of the polymorphism on the relationship between PAE and \%BF and found that the Arg allele did not have a significant impact on the relationship between physical activity and body composition.

Four weight reduction interventions consisting of exercise and diet have been conducted in Japan to date to assess whether the participants showed a significant difference in weight loss depending on the presence of the Trp64Arg polymorphism [10-12, 14]. Three of these studies found that subjects with the Arg allele had difficulty in losing body weight [10-12]. In two of these studies positive results were found by the same research group in a 3-month intervention for obese middle-aged women whose mean BMI was $>30 \mathrm{~kg} / \mathrm{m}^{2}[10,11]$; in the third study, also a 3-month intervention for middle-aged women, the differences in weight reduction was small $(-0.01$ vs. $-0.74 \mathrm{~kg}$ for subjects with and without the Arg allele, respectively) [12]. The most recent study followed middle-aged men and women who were enrolled in an intervention program for 6 months [14]. Both Arg allele carriers and non-carriers lost a significant amount of weight, but no significant 
difference was found with respect to Arg allele status ( -2.52 vs. $-1.89 \mathrm{~kg}$ for subjects with and without the Arg allele, respectively). An exercise + diet intervention study with obese Spanish women [13] obtained similar results to the most recent study involving a Japanese population. The results of these intervention studies are not consistent, and the discrepancies may be due to gender, body weight at baseline, duration of the study period, or the intensity of the intervention.

In an exercise-based intervention among white and black populations of both sexes, the authors estimated the impact of the polymorphism directly [7]. The intervention consisted of 20 weeks of ergometer training at 55-75\% $V \mathrm{O}_{2 \max }$ strength for 30-50 min, three times per week. The authors compared eight body composition items, including $\mathrm{BMI}$, body fat mass, and \% $\mathrm{BF}$, and observed no differences with respect to the Arg allele. A recent 12-week intervention conducted among Japanese males who were recommended to walk 10,000 steps/day obtained similar results [8]. Bea et al. [9] conducted a 1-year intervention study involving endurance training. Postmenopausal women randomized to the exercise group had a reduced $\% \mathrm{BF}$ irrespective of the presence of polymorphism, while control subjects with the Arg allele gained body fat compared to those without the Arg allele. We obtained a null result, even though we observed a population that was characterized by a wide range of PAE. The impact of the polymorphism on the association between exercise and body composition is therefore likely to be weak, if it exists at all.

There are a number of limitation to our study. First, we used bioelectrical impedance analysis (BIA) for the assessment of \%BF. Dual-energy X-ray absorptiometry (DXA) is now a standard method of assessing body composition, and BIA is considered to be less accurate. However, DXA involves exposure to X-rays. Thus, BIA seems to be favorable for studies of children. Moreover, BIA is easily applicable and is still used, even in recent studies [24-26]. We examined \% BF at approximately 3 p.m. to avoid the effects of diurnal variation. Second, the PAE values used in our study were not actually measured but obtained by questionnaire. While objective data on physical activity can be obtained by a triaxial accelerometer, the questionnaire was adapted to estimate participants' physical activity in the Framingham study [27]. Nearly all physical activity data on which all public health guidelines for physical activity are based derive from self-reports [28]. In addition, our PAE values were not an indicator of total physical activity but of sports or exercise. Here, we used $\mathrm{PAE}$ as a surrogate because participants were expected to have negligible energy expenditure due to labor. As well as the relationship between $\% \mathrm{BF}$ and physical activity measured by a triaxial accelerometer [18] or that between \% $\mathrm{BF}$ and physical activity-related energy expenditure [16, 17], $\mathrm{PAE}$ was shown to be significantly correlated with $\% \mathrm{BF}$, which supports the validity of our methodology. In a special population, as that of our study, PAE may be used as a proxy for total physical activity. Lastly, the small of study subjects is a limitation. Null results were obtained for interaction, which might be derived from the small sample size. Thus, the results may need to be interpreted with some caution.

In summary, when we examined young adult Japanese males with wide range of PAE, the Arg allele of the ADRB3 gene was found not to have a significant influence on the relationship between PAE and \%BF. Thus, the ADRB3 Trp64Arg polymorphism alone seems to have a limited impact, if it has any influence at all.

Acknowledgments The authors are grateful to Ms. Junko Aoyama for her technical assistance. This study was supported in part by a Grant-in-Aid for Scientific Research (10470098) from the Ministry of Education, Culture, Sports, Science and Technology of Japan to KO as well as by a research grant from the Kanae Foundation for the Promotion of Medical Science (1998) to TN.

Conflict of interest None.

\section{References}

1. Walston J, Silver K, Bogardus C, Knowler WC, Celi FS, Austin $\mathrm{S}$, et al. Time of onset of non-insulin-dependent diabetes mellitus and genetic variation in the beta 3-adrenergic-receptor gene. N Engl J Med. 1995;333:343-7.

2. Sipiläinen R, Uusitupa M, Heikkinen S, Rissanen A, Laakso M. Polymorphism of the beta3-adrenergic receptor gene affects basal metabolic rate in obese Finns. Diabetes. 1997;46:77-80.

3. Kadowaki H, Yasuda K, Iwamoto K, Otabe S, Shimokawa K, Silver K, et al. A mutation in the beta 3-adrenergic receptor gene is associated with obesity and hyperinsulinemia in Japanese subjects. Biochem Biophys Res Commun. 1995;215:555-60.

4. Nagase T, Aoki A, Yamamoto M, Yasuda H, Kado S, Nishikawa $\mathrm{M}$, et al. Lack of association between the Trp64Arg mutation in the beta 3-adrenergic receptor gene and obesity in Japanese men: a longitudinal analysis. J Clin Endocrinol Metab. 1997;82: 1284-7.

5. Kurokawa N, Nakai K, Kameo S, Liu ZM, Satoh H. Association of BMI with the beta3-adrenergic receptor gene polymorphism in Japanese: meta-analysis. Obes Res. 2001;9:741-5.

6. Kawaguchi H, Masuo K, Katsuya T, Sugimoto K, Rakugi H, Ogihara $\mathrm{T}$, et al. beta2- and beta3-Adrenoceptor polymorphisms relate to subsequent weight gain and blood pressure elevation in obese normotensive individuals. Hypertens Res. 2006;29:951-9.

7. Garenc C, Pérusse L, Rankinen T, Gagnon J, Leon AS, Skinner JS, et al. The Trp64Arg polymorphism of the beta3-adrenergic receptor gene is not associated with training-induced changes in body composition: the HERITAGE Family Study. Obes Res. 2001;9:337-41.

8. Tahara A, Osaki Y, Kishimoto T. Effect of the beta-3-adrenergic receptor gene polymorphism Trp64Arg on BMI reduction associated with an exercise based intervention program in Japanese middle-aged males. Environ Health Prev Med. 2010;15:392-7. 
9. Bea JW, Lohman TG, Cussler EC, Going SB, Thompson PA. Lifestyle modifies the relationship between body composition and adrenergic receptor genetic polymorphism, ADRB2, ADRB3 and ADRA2B: a secondary analysis of a randomized controlled trial of physical activity among postmenopausal women. Behav Genet. 2010;40:649-59.

10. Yoshida T, Sakane N, Umekawa T, Sakai M, Takahashi T, Kondo M. Mutation of $\beta 3$-adrenergic-receptor gene and response to treatment of obesity. Lancet. 1995;346:1433-4.

11. Sakane N, Yoshida T, Umekawa T, Kogure A, Takakura Y, Kondo M. Effects of Trp64Arg mutation in the $\beta 3$-adrenergic receptor gene on weight loss, body fat distribution, glycemic control, and insulin resistance in obese type 2 diabetic patients. Diabetes Care. 1997;20:1887-90.

12. Shiwaku K, Nogi A, Anuurad E, Kitajima K, Enkhmaa B, Shimono $\mathrm{K}$, et al. Difficulty in losing weight by behavioral intervention for women with Trp64Arg polymorphism of the $\beta 3$-adrenergic receptor gene. Int J Obes Relat Metab Disord. 2003;27:1028-36.

13. de Luis DA, Gonzalez Sagrado M, Aller R, Izaola O, Conde R. Influence of the Trp64Arg polymorphism in the beta 3 adrenoreceptor gene on insulin resistance, adipocytokine response, and weight loss secondary to lifestyle modification in obese patients. Eur J Intern Med. 2007;18:587-92.

14. Kuriyama S, Shimazu T, Hozawa A, Kure S, Kurokawa N, Kakizaki $\mathrm{M}$, et al. No effect of the Trp64Arg variant of the beta3-adrenergic receptor gene on weight loss by diet and exercise intervention among Japanese adults. Metabolism. 2008;57:1570-5.

15. Gourlan MJ, Trouilloud DO, Sarrazin PG. Interventions promoting physical activity among obese populations: a meta-analysis considering global effect, long-term maintenance, physical activity indicators and dose characteristics. Obes Rev. 2011;12:e633-45.

16. Westerterp KR, Goran MI. Relationship between physical activity related energy expenditure and body composition: a gender difference. Int J Obes Relat Metab Disord. 1997;21: $184-8$.

17. Paul DR, Novotony JA, Rumpler WV. Effects of the interaction of sex and food intake on the relation between energy expenditure and body composition. Am J Clin Nutr. 2004;79:385-9.
18. den Hoed M, Westerterp KR. Body composition is associated with physical activity in daily life as measured using a triaxial accelerometer in both men and women. Int J Obes. 2008;32: 1264-70.

19. Heyward VH. Gross energy expenditure for conditioning exercises, sports and recreational activities; in: advanced fitness assessment and exercise prescription. 6th ed. Champaign: Human Kinetics; 2010. p. 382-4.

20. Kraemer HC, Blasey CM. Centering in regression analyses: a strategy to prevent errors in statistical inference. Int J Methods Psychiatr Res. 2004;13:141-51.

21. Armitage P, Berry G, Matthews JSN (editors). Regression in groups. Statistical method in medical research. 4th ed. Malden: Blackwell Science. 2002. p. 322-30.

22. Armitage P, Berry G, Matthews JSN (editors). Analysis of covariance. Statistical method in medical research. 4th ed. Malden: Blackwell Science. 2002. p. 331-7.

23. Matsushita Y, Yokoyama T, Yoshiike Y, Matsumura Y, Date C, Kawahara K, et al. The Trp(64)Arg polymorphism of the beta(3)adrenergic receptor gene is not associated with body weight or body mass index in Japanese: a longitudinal analysis. J Clin Endocrinol Metab. 2003;88:5914-20.

24. Samani-Radia D, McCarthy HD. Comparison of children's body fatness between two contrasting income groups: contribution of height difference. Int J Obes (Lond). 2011;35:128-33.

25. Li SC, Liu YH, Liu JF, Chang WH, Chen CM, Chen CY. Almond consumption improved glycemic control and lipid profiles in patients with type 2 diabetes mellitus. Metabolism. 2011;60: 474-9.

26. Parkinson KN, Drewett RF, Jones AR, Dale A, Pearce MS, Wright CM, Gateshead Millennium Study Core Team, et al. When do mothers think their child is overweight? Int J Obes (Lond). 2011;35:510-6.

27. Sahni S, Hannan MT, Blumberg J, Cupples LA, Kiel DP, Tucker KL. Protective effect of total carotenoid and lycopene intake on the risk of hip fracture: a 17-year follow-up from the Framingham Osteoporosis Study. J Bone Miner Res. 2009;24:1086-94.

28. Haskell WL. Physical activity by self-report: a brief history and future issues. J Phys Act Health. 2012;9:S5-10. 